\title{
Improving lipoplex-mediated gene transfer into C6 glioma cells and primary neurons
}

\author{
M. Teresa Girão da Cruz, ${ }^{\mathrm{a}, \mathrm{b}}$ Sérgio Simões, ${ }^{\mathrm{b}, \mathrm{c}}$ and Maria C. Pedroso de Limaa ${ }^{\mathrm{a}, \mathrm{b}, *}$ \\ ${ }^{a}$ Department of Biochemistry, Faculty of Sciences and Technology, University of Coimbra, Apartado 3126, 3001-401 Coimbra, Portugal \\ ${ }^{\mathrm{b}}$ Center for Neuroscience and Cell Biology of Coimbra, University of Coimbra, 3004-517 Coimbra, Portugal \\ ${ }^{\mathrm{c}}$ Laboratory of Pharmaceutical Technology, Faculty of Pharmacy, University of Coimbra, 3000 Coimbra, Portugal
}

Received 22 July 2003; revised 11 December 2003; accepted 19 December 2003

\begin{abstract}
The development of methodologies for gene transfer into the central nervous system is crucial for gene therapy of neurological disorders. In this study, different cationic liposome formulations were used to transfer DNA into C6 glioma cells and primary hippocampal and cortical neurons by varying the nature of the helper lipid (DOPE, Chol) or a mixture of DOPE and cholesterol (Chol) associated to DOTAP. In addition, the effect of the lipid/DNA $(+/-)$ charge ratio, the association of the ligand transferrin to the lipoplexes, and the stage of differentiation of the primary cells on the levels of transfection activity, transfection efficiency, and duration of gene expression were evaluated. Mechanistic studies were also performed to investigate the route of delivery of the complexes into neurons. Our results indicate that DOTAP:Chol (1:1 mol ratio) was the best formulation to transfer a reporter gene into C6 glioma cells, primary hippocampal neurons, and primary cortical neurons. The use of transferrin-associated lipoplexes resulted in a significant enhancement of transfection activity, as compared to plain lipoplexes, which can be partially attributed to the promotion of their internalization mediated by transferrin. While for hippocampal neurons the levels of luciferase gene expression are very low, for primary cortical neurons the levels of transgene expression are high and relatively stable, although only $4 \%$ of the cells has been transfected. The stage of cell differentiation revealed to be critical to the levels of gene expression. Consistent with previous findings on the mechanisms of cell internalization, the experiments with inhibitors of the endocytotic pathway clearly indicate that transferrin-associated lipoplexes are internalized into primary neurons by endocytosis. Promising results were obtained in terms of the levels and duration of gene expression, particularly in cortical neurons when transfected with the Tfassociated lipoplexes, this finding suggesting the usefulness of these lipid-based carriers to deliver genes within the CNS.
\end{abstract}

(C) 2004 Elsevier Inc. All rights reserved.

Keywords: Cationic liposome; Lipoplex; Transferrin; C6 cells; Hippocampal neurons; Cortical neurons

\section{Introduction}

Gene transfer to cells of the CNS may represent a powerful tool to treat a large number of diseases, including neurological disorders. However, while some success has been reported regarding the application of gene therapy (SCID, cancer, etc.) (Aiuti et al., 2002; Cavazzana-Calvo et al., 2000; Reid et al., 2002), its use to the CNS has been hindered by the inefficient transfection of postmitotic cells.

Two main approaches are available for gene transfer into CNS: the ex vivo approach involving the genetic manipu-

\footnotetext{
* Corresponding author. Department of Biochemistry, Faculty of Sciences and Technology, University of Coimbra, Apartado 3126, 3001401 Coimbra, Portugal. Fax: +351-239-853-607.

E-mail address: mdelima@ci.uc.pt (M.C. Pedroso de Lima).
}

lation of cells followed by their grafting into the brain, this way circumventing the blood-brain barrier; and the in vivo approach involving the local gene transfer to CNS cells (stereotactic administration using either a viral or a nonviral vector). Work in this area has been mainly based on the use of both adenoviral and lentiviral vectors (Berry et al., 2001; Clark et al., 1999; Hughes et al., 2002; King et al., 2000; Martino et al., 1998), largely due to their high gene transfer efficiency and neuronal tropism. Nevertheless, important safety limitations have been associated to viral vectors, including the risk of immunogenicity, cytotoxicity, and tumorigenicity.

Nonviral vectors exhibit some technological, therapeutic, and safety advantages and may therefore constitute a viable alternative to viral vectors aiming at applying gene therapy strategies to CNS. Nonviral vectors include naked DNA, 
gene gun, cationic polymers, and cationic liposomes. The injection of naked DNA has been reported for glia and neurons in the mouse brain (Garcia and Sharma, 1998; Schwartz et al., 1996), although levels of transgene expression have been reported to be very low. This method may be improved if a "bio-ballistic" gene gun is used to force small gold particles coated with plasmid DNA to enter into cells (Sato et al., 2000; Thomas et al., 1998), although optimization is required to prevent the damage of neuronal tissue (Hui et al., 1994). Polycationic polymers, such as poly-Llysine or polyethylenimine (PEI), have also been used to condense plasmid DNA (forming polyplexes) aiming at neuronal transfection (Goula et al., 1998; Lemkine et al., 1999). However, the duration of expression is very short, even if high levels of transgene expression have been achieved. A large amount of effort has also been devoted to the development of cationic liposomes (Imaoka et al., 1998; Murray et al., 1999; Roessler and Davidson, 1994; Wu et al., 2000; Zou et al., 1999), the most widely used nonviral vectors, to improve transfection efficiency and duration of gene expression as well as to confer adequate features for their in vivo use. The ability of this system to mediate transfection was attributed to recognition of certain properties, namely, (1) a spontaneous electrostatic interaction between the positively charged liposomes and the negatively charged DNA, which results in an efficient condensation of the nucleic acids, and (2) the fact that the resulting cationic liposome/DNA complexes (lipoplexes) could exhibit a net positive charge that promotes their association with the negatively charged cell surface (da Cruz et al., 2001; Pires et al., 1999). Following internalization by endocytosis (Gao and Huang, 1995; Zabner et al., 1995), the lipoplexes promote the cytoplasmic delivery of DNA (Felgner, 1996; Felgner et al., 1987; Gao and Huang, 1995; Roessler and Davidson, 1994; Zhu et al., 1993). Cationic lipids have been used as neuronal gene delivery agents, and some promising studies were reported (Roessler and Davidson, 1994; Zou et al., 1999), namely for liposomes composed of DC-Chol (Zou et al., 1999). Nevertheless, the low efficiency of expression mediated by such systems, as compared to viral vectors, clearly indicates that additional work is needed to perfect their design to make them more than an experimental concept for the therapy of neurological disorders.

A variety of mono- or multivalent cationic lipids are currently available for gene transfer (e.g., DC-Chol, DOTMA, DOSPA, DOGS, and DOTAP) (Gao and Huang, 1991; Imaoka et al., 1998; Kofler et al., 1998; Zou et al., 1999). In most cases, these lipids are mixed with an equimolar amount of a "helper" lipid. Most frequently, the neutral lipid DOPE is used, since this is known to enhance transfection efficiency (Felgner et al., 1994; Gao and Huang, 1995; Stegmann and Legendre, 1997; Zhou and Huang, 1994), due to its ability to facilitate the formation of cationic liposomes and to its tendency to form non-bilayer phases that may contribute to the destabilization of the endosomal membrane (Farhood et al., 1995; Felgner et al., 1994; Legendre and Szoka, 1992; Zhou and Huang, 1994). On the other hand, cholesterol (Chol) constitutes another promising "helper" lipid, since it has been demonstrated that high levels of transfection can be obtained both in vitro and in vivo, upon its association with a cationic lipid (Crook et al., 1998; Templeton et al., 1997). Moreover, the inclusion of Chol can potentially reduce the destabilization of lipoplexes in the presence of serum, thus increasing the biological stability of such lipoplexes.

Aiming at enhancing transfection activity mediated by cationic liposomes, different strategies have been tried, including the association of targeting ligands (Cheng, 1996; Simoes et al., 1998, 1999b), fusogenic proteins (Simoes et al., 2000), and fusogenic (Kichler et al., 1997; Plank et al., 1994) and NLS (Chan and Jans, 1999; Zanta et al., 1999) peptides. Targeting DNA vectors to cellular Tf receptors has been tried successfully before by linking the ligand to polycation conjugates (Liu et al., 1995; Zhu et al., 1993), through association of the protein with cationic liposomes and by covalent coupling of anti-Tf receptor monoclonal antibodies to the distal ends of PEG on sterically stabilized liposomes (Shi et al., 2001) or on nanoparticles (Olivier et al., 2002). In this regard, we and others have demonstrated that the association of human $\mathrm{Tf}$ to cationic liposome/DNA complexes enhances transfection in a large variety of cells, including dividing and nondividing cells (Cheng, 1996; Simoes et al., 1998, 1999b).

The aim of the present study was to evaluate whether association of iron-saturated human $\mathrm{Tf}$ to cationic liposome/ DNA complexes would result in high levels of transfection in a rodent glioma cell line and in rodent neuronal primary cultures (hippocampal and cortical). Besides formulation development, the effect of different variables on the duration and levels of gene expression was evaluated to assess the optimal transfection parameters for in vivo or ex vivo use.

\section{Materials and methods}

Cells

\section{Cell lines}

C6 cells, an adherent fibroblast cell line derived from rat tissue (glioma), were obtained from American Type Culture Collection, MD, USA. Cells were incubated at $37^{\circ} \mathrm{C}$ in a humidified atmosphere containing $5 \% \mathrm{CO}_{2}$ and maintained in Ham's F10 medium (Sigma, St. Louis, MO, USA) supplemented with $15 \%$ heat-inactivated horse serum (HS) (Gibco, Paisley, Scotland), 2.5\% heat-inactivated fetal calf serum (FCS) (Biochrom KG, Berlin, Germany), $1.168 \mathrm{~g}$ glutamine $/ 1,100 \mu \mathrm{g} / \mathrm{ml}$ of streptomycin, and $1 \mathrm{unit} / \mathrm{ml}$ of penicillin (Sigma). Cells were propagated by diluting the cell suspension $1 / 2$ to $1 / 3$ every $3-4$ days. For use in transfection experiments, C6 cells were plated in 48-well plates (Corning Costar Corporation, Cambridge, MA, 
USA), at a cell density of $0.1 \times 10^{6}$ cells/well, in a final volume of $1 \mathrm{ml}, 1$ day before incubation with lipoplexes. Cell viability was determined by Trypan blue exclusion.

\section{Primary cell cultures}

Hippocampal neurons. Hippocampi were dissected from the brains of 18- or 19-day gestation Wistar rat fetuses (Banker and Cowan, 1977). After dissociation and centrifugation, the tissue was resuspended in serum-free Neurobasal medium (Gibco), enriched with $2 \%$ v/v B27 supplement (Brewer et al., 1993), 0.005\% gentamicin (Gibco), $0.05 \%$ glutamine, and $25 \mu \mathrm{M}$ glutamate (Sigma). Cells were plated at $0.1 \times 10^{6}$ cells/well on 48 -well plates, or $0.1 \times 10^{6}$ cells/coverslip (16-mm-diameter), previously coated with poly-L-lysine. Cultures were kept at $37^{\circ} \mathrm{C}$ in a humidified atmosphere containing $5 \% \mathrm{CO}_{2}$. Cells were used for transfection experiments after 6 days in culture.

Cortical neurons. Cortices were dissected from the brains of 15- or 16-day gestation Wistar rat fetuses (Hertz et al., 1989). After dissociation and centrifugation, the tissue was resuspended in Neurobasal medium, enriched with 2\% B27 supplement, $0.2 \%$ glutamine, $100 \mu \mathrm{g} / \mathrm{ml}$ of streptomycin, and $1 \mathrm{unit} / \mathrm{ml}$ of penicillin. Cells were plated at $0.1 \times 10^{6}$ cells/well on 48 -well plates, or $0.1 \times 10^{6}$ cells/coverslip (16-mm-diameter), previously coated with poly-L-lysine. Cultures were kept at $37^{\circ} \mathrm{C}$ in a humidified atmosphere containing $5 \% \mathrm{CO}_{2}$. Cells were used for transfection experiments after 6 days in culture.

\section{Preparation of cationic liposomes and lipoplexes}

The lipids 1,2-dioleoyl-3-trimethylammonium-propane (DOTAP) and L- $\alpha$-dioleoyl-phosphatidylethanolamine (DOPE) were purchased from Avanti Polar Lipids (Alabaster, AL, USA). Chol was purchased from Sigma. Liposomes containing DOTAP:DOPE, DOTAP:Chol, or DOTAP: DOPE:Chol at 1:1, 1:1, and 2:1:1 mol ratios, respectively, were prepared by mixing the lipids and drying them from chloroform solution under vacuum using a rotatory evaporator. The dried lipid film was rehydrated with distilled water to a DOTAP concentration of $2.5 \mathrm{mg} / \mathrm{ml}$ and sonicated for 5 $\min$. The resulting liposomes were then extruded 21 times through two stacked polycarbonate membranes $(50-\mathrm{nm}$ pore diameter) and diluted with distilled water to a final DOTAP concentration of $1 \mathrm{mg} / \mathrm{ml}$. The suspension was stored at $4^{\circ} \mathrm{C}$, under nitrogen, until use. Phospholipid concentration was determined by a phosphate assay (Bartlett, 1959). Chol concentration was measured with Sigma Diagnostics ${ }^{\circledR}$ Cholesterol Reagent (Sigma). Plain lipoplexes were prepared by sequentially mixing $100 \mu \mathrm{l}$ of a HEPES-buffered saline solution (20 mM HEPES, $100 \mathrm{mM} \mathrm{NaCl}$, pH 7.4, Sigma) to a predetermined volume of liposomes composed of DOTAP:DOPE, DOTAP:Chol, or DOTAP:DOPE:Chol to obtain the desired lipid/DNA (+/-) charge ratio, $15 \mathrm{~min}$ before the addition of $100 \mu \mathrm{l}$ of DNA solution, containing 1 $\mu \mathrm{g}$ of plasmid dissolved in distilled water. The mixture was incubated for $15 \mathrm{~min}$ at room temperature before addition to the cells. The Tf-lipoplexes were obtained by gently mixing $100 \mu \mathrm{l}$ of human Tf solution (Sigma) to a predetermined volume of liposomes, $15 \mathrm{~min}$ before the addition of 100 $\mu \mathrm{l}$ DNA solution, and the resulting mixture was further incubated for $15 \mathrm{~min}$. The Tf solution was prepared at 320 $\mu \mathrm{g} / \mathrm{ml}$ in a HEPES-buffered saline solution (20 mM HEPES, $100 \mathrm{mM} \mathrm{NaCl}, \mathrm{pH}$ 7.4, Sigma). Lipoplexes were used immediately after being prepared.

\section{Cationic lipid-mediated transfection}

\section{Transfection activity}

The transfection activity of lipoplexes (plain and Tfassociated) was evaluated by measuring luciferase gene expression levels (pCMVluc plasmid). Cells were rinsed twice with serum-free medium and covered with $0.3 \mathrm{ml}$ of medium. Lipoplexes were added to cells in a volume of approximately $0.2 \mathrm{ml}$ per well. After $4 \mathrm{~h}$ of incubation (in $5 \% \mathrm{CO}_{2}$ at $37^{\circ} \mathrm{C}$ ), the medium was replaced and the cells were further incubated for different periods of time. The cells were then washed twice with phosphate-buffered saline (PBS), and $100 \mu \mathrm{l}$ of lyses buffer (1 mM DTT; 1 mM EDTA; 25 mM Tris-phosphate, $\mathrm{pH}$ 7.8; $8 \mathrm{mM} \mathrm{MgCl}_{2}$; $15 \%$ glycerol; $1 \% \mathrm{v} / \mathrm{v}$ Triton X-100 [Sigma]) was added to each well. The levels of gene expression in the lysates were evaluated by luminescence measurements using a

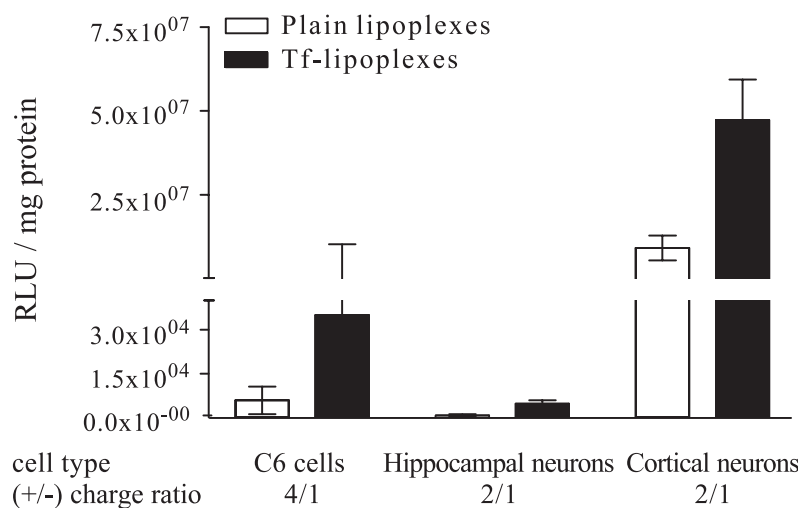

Fig. 1. Effect of associating transferrin (Tf) to DOTAP:Chol liposomes on luciferase gene expression in C6 cells and in primary hippocampal and cortical neurons. C6 cells were plated 1 day before transfection, and primary cells were maintained in culture for 6 days before transfection. The liposomes were complexed, in the absence (plain lipoplexes) or in the presence (Tflipoplexes) of $32 \mu \mathrm{g}$ of Tf, with $1 \mu \mathrm{g}$ of pCMVluc at the indicated lipid/DNA $(+/-)$ charge ratios and added to the cells, in the absence of serum. After incubation for $4 \mathrm{~h}$, the medium was replaced and the cells were further incubated for $12 \mathrm{~h}$ (C6 cells; hippocampal neurons) or $24 \mathrm{~h}$ (cortical neurons). The levels of gene expression were evaluated by luminescence measurements and the data are expressed as RLU per milligram of total cell protein (mean \pm standard deviation obtained from triplicate wells and representative of two independent experiments). 


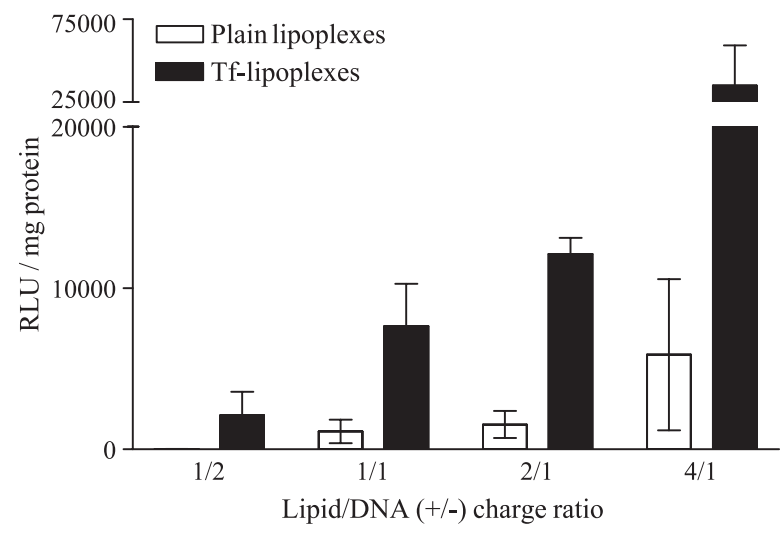

Fig. 2. Effect of lipid/DNA (+/-) charge ratio on transfection of C6 cells mediated by plain and Tf-associated lipoplexes. Cells were plated 1 day before transfection. DOTAP:Chol liposomes were complexed, in the absence (plain lipoplexes) or in the presence (Tf-lipoplexes) of $32 \mu \mathrm{g}$ of Tf, with $1 \mu \mathrm{g}$ of pCMVluc at the indicated lipid/DNA (+/-) charge ratios and added to the cells, in the absence of serum. After incubation for $4 \mathrm{~h}$, the medium was replaced and the cells were further incubated for $12 \mathrm{~h}$. The levels of gene expression were evaluated by luminescence measurements and the data are expressed as RLU per milligram of total cell protein (mean \pm standard deviation obtained from triplicate wells and representative of two independent experiments).

luminometer (Mediators Diagnostics GmbH, Wien, Austria). The protein content of the lysates was measured by the Sedmak method (Sedmak and Grossberg, 1977) using bovine serum albumin (BSA) as the standard. The data were expressed as relative light units (RLU) per milligrams of total cell protein. Following incubation of cells with lipoplexes for different periods of time, cytotoxicity mediated by the lipoplexes was measured by the Alamar blue assay (Fields and Lancaster, 1993; White et al., 1996)

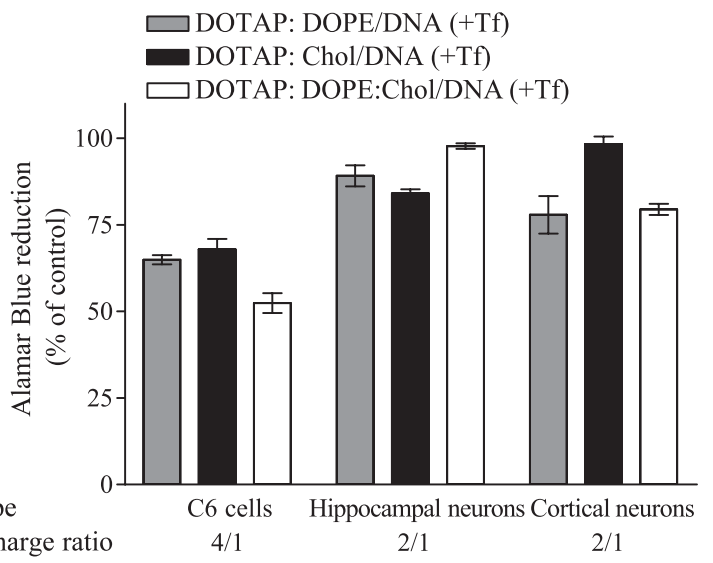

Fig. 3. Cytotoxicity of Tf-lipoplexes prepared from DOTAP:DOPE, DOTAP:Chol, and DOTAP:DOPE:Chol liposomes. C6 cells were plated 1 day before transfection; hippocampal and cortical neurons were maintained in culture for 6 days before transfection. Transfection was performed as described in Fig. 1. Cytotoxicity was evaluated by the Alamar blue reduction assay, as described in the Materials and methods. The data are expressed as a percentage of control (nontransfected cells). Results represent the mean \pm standard deviation obtained from triplicate wells and representative of two independent experiments.

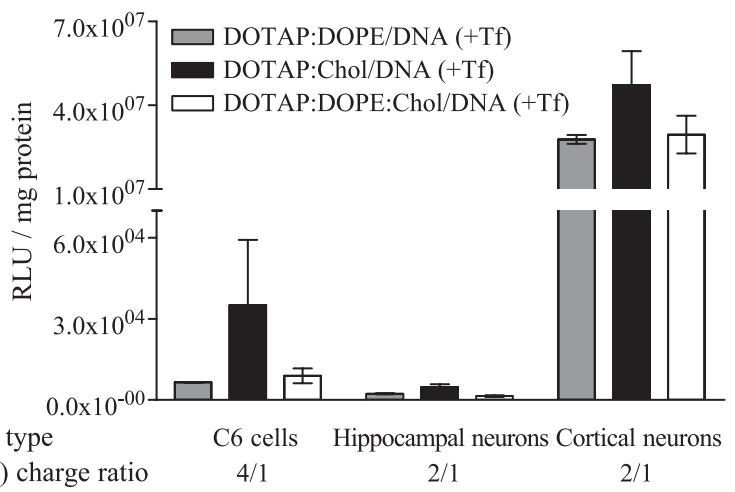

Fig. 4. Effect of liposome composition on luciferase gene expression in C6 cells and in primary hippocampal and cortical neurons. C6 cells were plated 1 day before transfection; primary cells were maintained in culture for 6 days before transfection. DOTAP:DOPE, DOTAP:Chol, and DOTAP: DOPE:Chol liposomes were complexed, in the presence of $32 \mu \mathrm{g}$ of $\mathrm{Tf}$, with $1 \mu \mathrm{g}$ of pCMVluc at the indicated lipid/DNA $(+/-)$ charge ratios. Transfection was performed as described in Fig. 1. Data are expressed as RLU per milligram of total cell protein (mean \pm standard deviation obtained from triplicate wells and representative of two independent experiments).
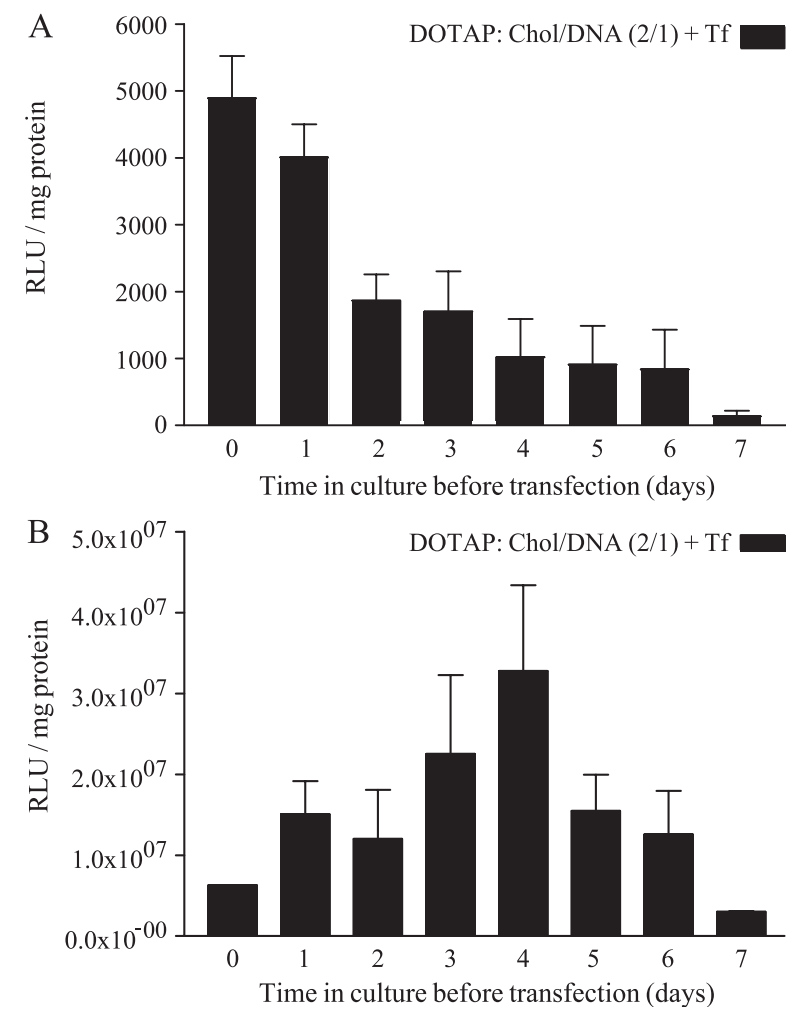

Fig. 5. Effect of the neuronal stage of differentiation on luciferase gene expression. Hippocampal (A) and cortical (B) neurons were maintained in culture $0-7$ days before transfection. DOTAP:Chol liposomes were complexed, in the presence of $32 \mu \mathrm{g}$ of Tf, with $1 \mu \mathrm{g}$ of pCMVluc at $2 / 1$ lipid/DNA (+/-) charge ratio and added to the cells. After incubation for 4 $\mathrm{h}$, the medium was replaced and the cells were further incubated for $12 \mathrm{~h}(\mathrm{~A})$ or $24 \mathrm{~h}$ (B). The levels of gene expression were evaluated by luminescence measurements and the data are expressed as RLU per milligram of total cell protein (mean \pm standard deviation obtained from triplicate wells and representative of two independent experiments). 
(Accurate Chemical and Scientific Corporation, Westbury, NY, USA).

\section{Transfection efficiency}

The transfection efficiency of lipoplexes was determined by scoring cells expressing green fluorescent protein (pEGFP plasmid). Cells transfected with $1 \mu \mathrm{g}$ of pEGFP (as in previous section) were washed with PBS and fixed in a solution of 4\% paraformaldehyde (BKK Riedel-de Haën, Seelze, Germany) and 4\% sucrose (E. Merck, Darmstadt, Germany) in PBS. The cells plated on coverslips were rinsed in PBS and stained with DAPI (Molecular Probes, Leiden, The Netherlands). After further rinsing, the coverslips were mounted using the Prolong ${ }^{\circledR}$ Antifade kit (Molecular Probes). The samples were observed using a fluorescence microscope (Nikon, Diaphot-TMD, Tokyo, Japan) equipped with the appropriate filter (XF-63, Omega Optical Inc., Brattleboro, VT, USA). The percentage of cells expressing GFP was evaluated by counting cells in 10 randomly selected areas per slide.

\section{Immunocytochemical staining}

Neuron cultures, fixed for 30 min with $4 \%$ paraformaldehyde and $4 \%$ sucrose in PBS, were permeabilized with $0.2 \%$ Triton X-100 (E. Merck, Darmstadt, Germany) and blocked with 3\% BSA (Sigma) for $30 \mathrm{~min}$. Cells were then incubated for $1 \mathrm{~h}$ with primary antibody anti-MAP2 (Sigma; dilution 1:500), an antibody that binds to the cytoskeletal microtubule-associated protein 2 (MAP2) primarily expressed in the somatodendritic domain of neurons but

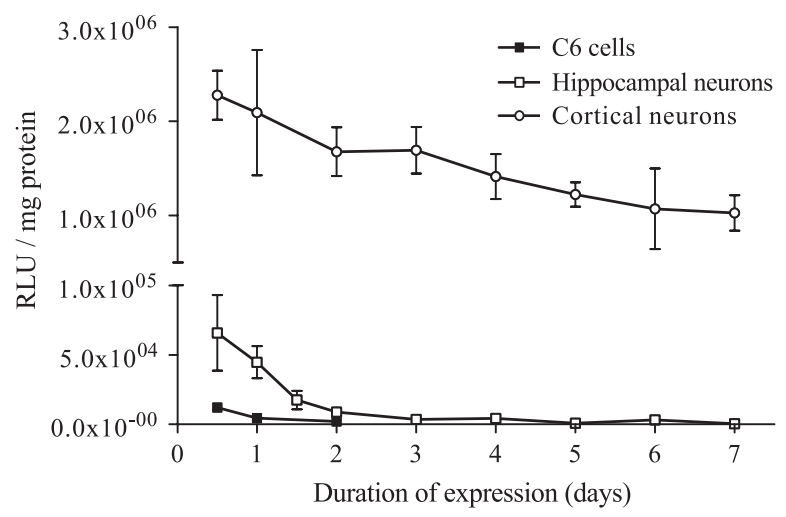

Fig. 6. Duration of gene expression upon transfection of C6 cells and primary hippocampal and cortical neurons. C6 cells were plated 1 day before transfection, and primary hippocampal and cortical neurons were maintained in culture for 1 or 4 days, respectively, before transfection. DOTAP:Chol liposomes were complexed, in the presence of $32 \mu \mathrm{g}$ of $\mathrm{Tf}$, with $1 \mu \mathrm{g}$ of pCMVluc at 2/1 lipid/DNA (+/-) charge ratio and added to the cells, in the absence of serum. After incubation for $4 \mathrm{~h}$, the medium was replaced and the cells were further incubated for different time periods. The levels of gene expression were evaluated by luminescence measurements and the data are expressed as RLU per milligram of total cell protein (mean \pm standard deviation obtained from triplicate wells and representative of two independent experiments).
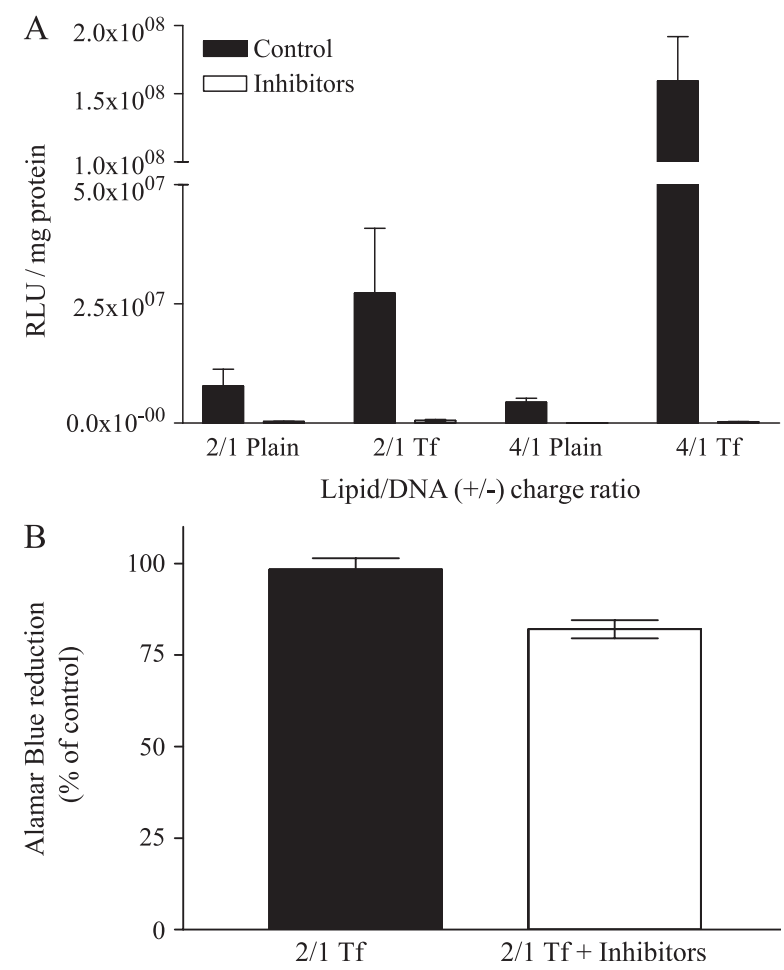

Fig. 7. Effect of inhibitors of endocytosis on luciferase gene expression (A) and on viability (B) of primary cortical neurons. Primary cortical neurons were maintained in culture for 4 days before transfection. Cells were pretreated with inhibitors of endocytosis $(1 \mu \mathrm{g} / \mathrm{ml}$ of antimycin A, $10 \mathrm{mM}$ $\mathrm{NaF}, 0.1 \%(\mathrm{w} / \mathrm{v})$ sodium azide) for $30 \mathrm{~min}$ at $37^{\circ} \mathrm{C}$ before incubation with the complexes. DOTAP:Chol liposomes were complexed, in the presence or the absence of $32 \mu \mathrm{g}$ of Tf, with $1 \mu \mathrm{g}$ of pCMVluc at $2 / 1$ or $4 / 1 \mathrm{lipid} / \mathrm{DNA}$ $(+/-)$ charge ratios and added to the treated or untreated (control) cells. After incubation for $1 \mathrm{~h}$, the medium was replaced and the cells were further incubated for $24 \mathrm{~h}$. (A) The levels of gene expression were evaluated by luminescence measurements and the data are expressed as RLU per milligram of total cell protein (mean \pm standard deviation obtained from triplicate wells and representative of two independent experiments). (B) Cytotoxicity was evaluated by the Alamar blue reduction assay, as described in the Materials and methods. The data are expressed as a percentage of the control (nontransfected cells). Results represent the mean \pm standard deviation obtained from triplicate wells and are representative of two independent experiments.

also to a lesser extent in glia; or anti-GFAP (Sigma; dilution 1:200), an antibody that is directed against the glial fibrillary acidic protein (GFAP), the main subunit of intermediate filaments of astrocytes in the CNS; or anti-CD11b (Serotec, Oxford, UK; dilution 1:100), an antibody that binds to the CD11b-antigen located on the lymphocyte function-associated antigen-1 and used here to identify microglia. Alexa Fluor ${ }^{\circledR}$ 488- or Alexa Fluor ${ }^{\circledR}$ 594-conjugated secondary antibodies (Molecular Probes) were applied at 1:200 for $1 \mathrm{~h}$. After further rinsing, the coverslips were mounted using the Prolong ${ }^{\circledR}$ Antifade kit (Molecular Probes). The slides were observed using a fluorescence microscope (Nikon, DiaphotTMD, Tokyo, Japan) equipped with the appropriate filters (605DF55, Nikon, Tokyo, Japan; XF-63 NS514, and 535DF45, Omega Optical Inc.). 


\section{Results}

Effect of associating transferrin to the complexes and of their charge ratio on transfection activity and cytotoxicity

It has been demonstrated that $\mathrm{Tf}$ is highly effective in promoting lipoplex-mediated transfection of various cells lines and primary cell cultures (de Lima et al., 1999; Simoes et al., 1998, 1999a,b,c; Wagner et al., 1994). Therefore, in this work, we evaluated whether this strategy would result in an enhancement of transfection of neuronal cells. The association of $\mathrm{Tf}$ to lipoplexes resulted in a significant enhancement of luciferase gene expression in C6 cells, where a 170-fold increase was observed as compared to plain lipoplexes, as well as in primary hippocampal and cortical neurons (600- and 50-fold increase, respectively). Fig. 1 illustrates the results obtained at lipid/DNA charge ratios that led to the highest levels of transgene expression for each type of transfected cells. This enhancing effect of Tf was more crucial for the hippocampal neurons for which the lowest values of transfection activity were observed.

Regarding the effect of charge ratio on transfection activity, a different behavior was observed depending on the cell type. For C6 cells (Fig. 2), the highest values of transfection were observed for the positively charged complexes (prepared at a 4/1 lipid/DNA charge ratio), while for primary neuronal cultures, neutral complexes $(2: 1$ charge ratio) were the most efficient (data not shown). As assessed by the Alamar blue assay, upon incubation of the cells with Tf-lipoplexes prepared at the optimal lipid/DNA charge ratio, a $25 \%$ reduction in viability was observed for C6 glioma cells, while for the primary cultures, the toxic effect was less pronounced $(10-15 \%)$ (Fig. 3). Overall, these results suggest that caution should be taken when such vectors (at the optimal transfection conditions) are used to mediate gene delivery.

\section{Influence of liposome composition on transfection activity}

Different liposome compositions were evaluated aiming at further improving lipoplex-mediated transfection of brain cells. For this purpose, Tf-lipoplexes composed of DOTAP: DOPE, DOTAP:Chol, or DOTAP:DOPE:Chol and DNA

Fig. 8. (A) Fluorescence and (B) bright field microscopy images of primary cortical neurons stained with DAPI and transfected with a plasmid coding for GFP. (C) Transfection efficiency of Tf-lipoplexes in primary hippocampal and cortical neurons. Cells were maintained in culture for 6 days before transfection. DOTAP:Chol liposomes were complexed, in the presence of $32 \mu \mathrm{g}$ of Tf, with $1 \mu \mathrm{g}$ of pEGFP at $2 / 1$ lipid/DNA $(+/-)$ charge ratio and added to the cells. After incubation for $4 \mathrm{~h}$, the medium was replaced and the cells were further incubated for $24 \mathrm{~h}$. The cells were fixed, stained as described in the Materials and methods, and photographed using a fluorescence microscope (Nikon, Diaphot-TMD) with the appropriate filters (XF-63 NS514, and 535DF45, Omega Optical Inc.) equipped with a camera (Nikon F-301). Scale bar represents $2.1 \mu \mathrm{m}$. (C) The percentage of cells expressing GFP was evaluated by counting cells in 10 randomly selected areas per slide. were prepared at different lipid/DNA (+/-) charge ratios and incubated with cells. As illustrated in Fig. 4, DOTAP: Chol/DNA lipoplexes were the most efficient to transfect C6 cells, and both primary hippocampal and cortical neurons. This enhancing effect can be attributed to the inclusion of Chol as a helper lipid in liposomes, as already reported by others (Crook et al., 1998; Hafez et al., 2001).

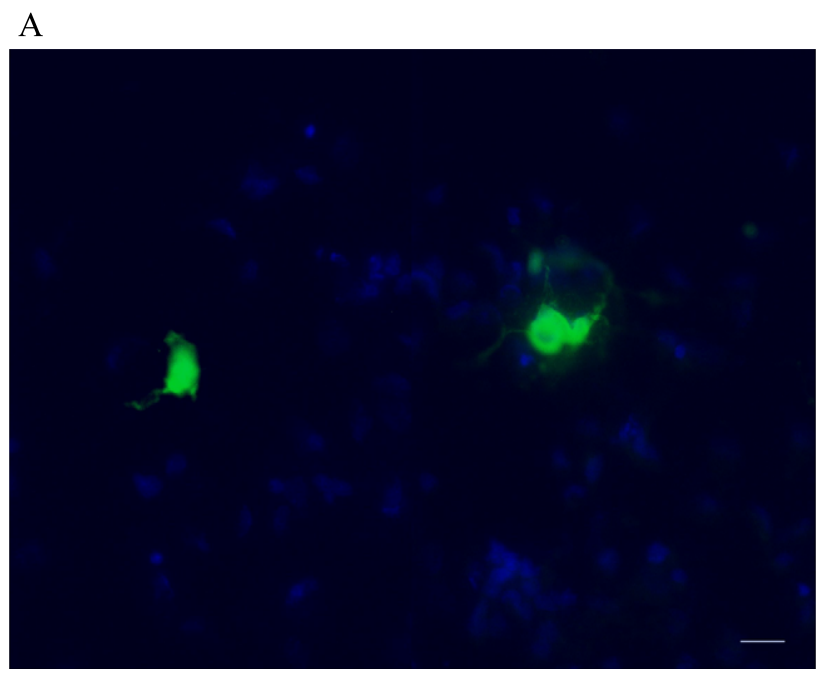

B

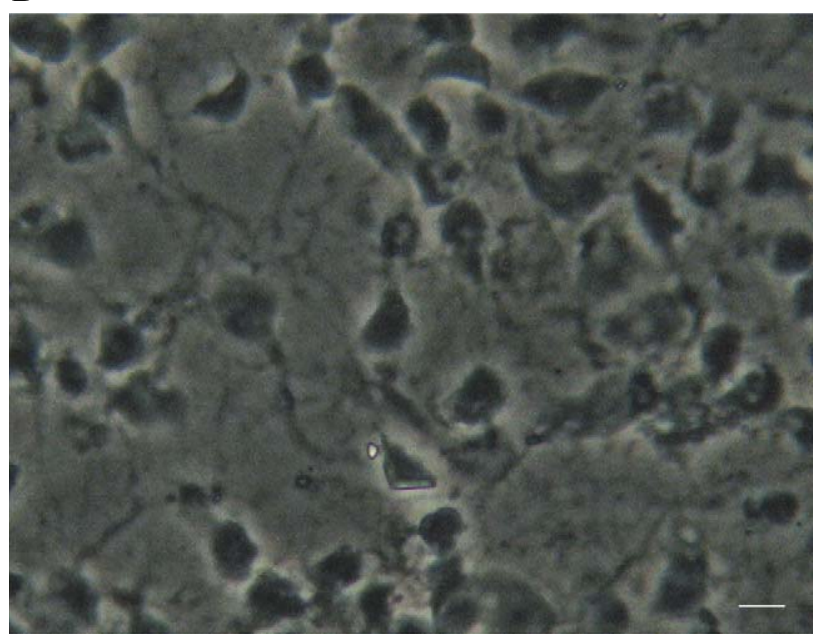

$\mathrm{C}$

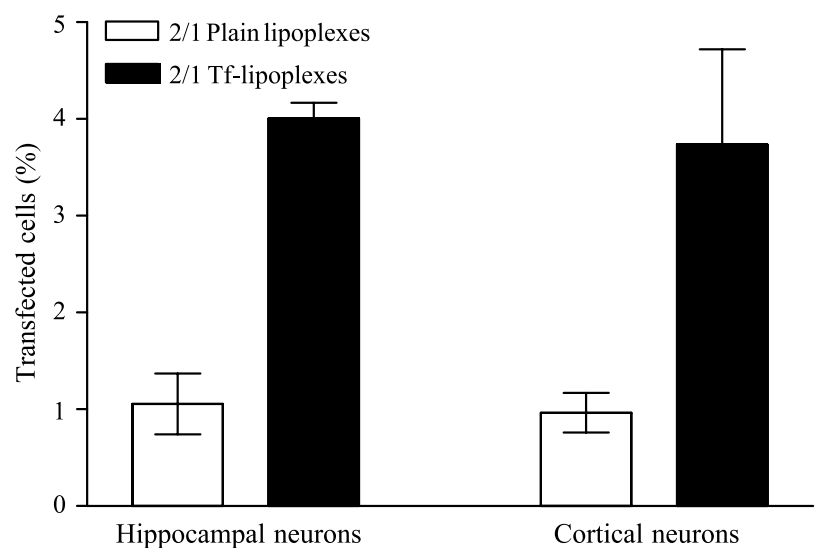


Besides improving the stability of liposomes, the presence of Chol reduces the binding of serum proteins to the lipoplexes, thus improving their biological stability (Faneca et al., 2002).

\section{Effect of cell differentiation time on transfection activity}

The stage of differentiation of primary cultures has been shown to affect cell biological behavior, including the ability to undergo endocytosis and the transcription activity. Therefore, we evaluated the importance of the stage of differentiation of neurons in transfection activity, by incubating cells with lipoplexes at different times after being plated. Luciferase expression was evaluated 12 and 24 $\mathrm{h}$ following transfection of hippocampal and cortical neurons, respectively. As shown in Fig. 5A, for hippocampal neurons, from 0 - to 7-day-old, a significant decrease in the levels of transgene expression was observed when ternary complexes composed of DOTAP:Chol and prepared at the 2/1 lipid/DNA (+/-) charge ratio were used. For cortical neurons (Fig. 5B), a different pattern was observed, the highest levels of luciferase expression being achieved for 4day-old cells. Nevertheless, a significant decrease was found for longer differentiation times. The low levels of endocytotic activity in highly differentiated and quiescent cells may result in a reduced internalization of lipoplexes, thus leading to the decrease of transgene expression.

\section{Duration of transgene expression}

The time dependence of gene expression was investigated by maintaining the cells in culture for different times after being transfected. As illustrated in Fig. 6, the levels of transgene expression significantly decreased for both C6 cells and hippocampal neurons, being essentially abolished at 2 days post-transfection. These results clearly indicate that using this transfection protocol, a transient expression was achieved. Nevertheless, for cortical neurons, only a slight decrease in transfection activity was observed over time, indicating that a relatively stable gene expression pattern was achieved for the time period tested.

\section{Effect of inhibitors of the endocytotic pathway on transfection activity}

To clarify the mechanisms involved in the internalization of the complexes by neuronal cells, we carried out experiments to assess transfection activity in the presence of chemical agents that interfere with the endocytotic pathway. For that purpose, cortical cells were pretreated for $30 \mathrm{~min}$ with a mixture of antimycin $\mathrm{A}, \mathrm{NaF}$, and $\mathrm{NaN}_{3}$ (Lee et al., 1993; Slepushkin et al., 1997), which strongly inhibits both receptor- and non-receptor-mediated endocytosis, before incubation with the complexes. Under these conditions, an inhibitory effect on transfection was evident both for plain
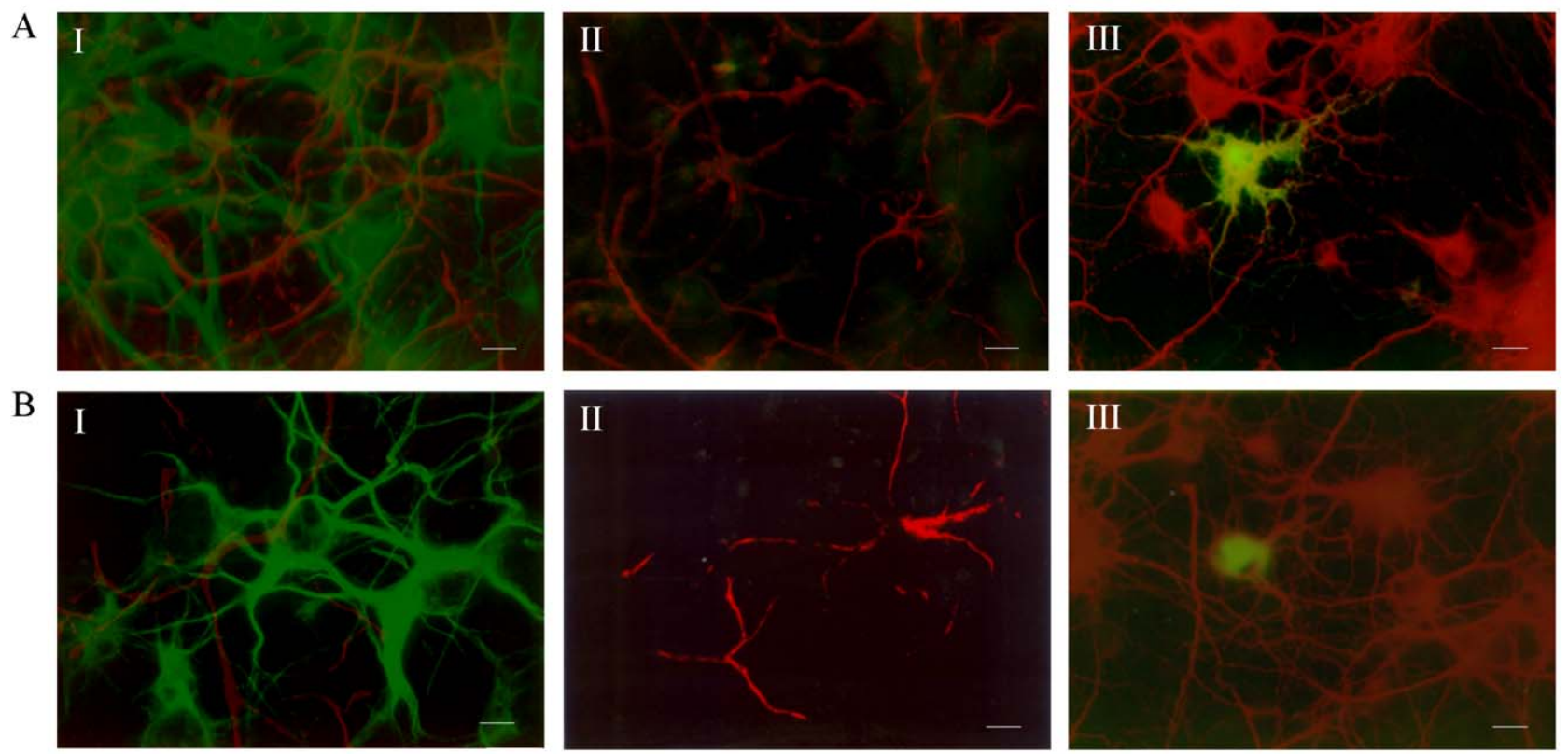

Fig. 9. Fluorescence microscopy images of hippocampal (A) and cortical (B) cultures immunocytochemically labeled for MAP2, GFAP, and CD11b and transfected with a plasmid coding for GFP. Cells were maintained in culture for 6 days before transfection. DOTAP:Chol liposomes were complexed, in the presence of $32 \mu \mathrm{g}$ of Tf, with $1 \mu \mathrm{g}$ of pEGFP at 2/1 lipid/DNA (+/-) charge ratio and added to the cells. After incubation for $4 \mathrm{~h}$, the medium was replaced and the cells were further incubated for 12, 24, and $48 \mathrm{~h}$. The cells were fixed and the immunocytochemistry was performed as described in Materials and methods. Cells were observed under a fluorescence microscope (Nikon, Diaphot-TMD) equipped with the appropriate filters (605DF55, Nikon; XF-63 NS514, and 535DF45, Omega Optical Inc.) equipped with a camera (Nikon F-301). Scale bar represents $2.1 \mu \mathrm{m}$. (I) Control; anti-MAP2 (green) and anti-GFAP (red) double labeling; (II) control; anti-CD11b (green) and anti-GFAP (red) double labeling; (III) anti-MAP2 labeling (red); GFP ${ }^{+}$neuron (yellow-colocalization). 
and ternary complexes, independently of their charge ratio (Fig. 7A). This fact reinforces that endocytosis is the main pathway for the uptake of lipoplexes (prepared with or without Tf), in accordance with previous findings (Friend et al., 1996; Simoes et al., 1999b; Xu and Szoka, 1996; Zhou and Huang, 1994). It should be noted that pretreatment of the cells with the inhibitors of endocytosis did not significantly affect their viability (Fig. 7B), which reinforces the reliability of these findings. Experiments involving the treatment of hippocampal neurons with inhibitors of endocytosis were also performed, but due to the low levels obtained for the gene expression even for the control condition (without inhibitors of endocytosis), no inhibitory effect was detected.

\section{Scoring and characterization of transfected cells}

In addition to evaluate the transfection activity (assessed by quantitating luciferase gene expression), transfected cells were scored upon evaluation of GFP expression. The number of successfully transfected hippocampal and cortical neurons was evaluated using fluorescence microscopy as described in the Materials and methods. The percentage of cells that expressed the transgene was very low for both cell types. Nevertheless, it was evident that ternary complexes (containing $\mathrm{Tf}$ ) were much more effective than plain lipoplexes, leading to an increase up to $4 \%$ of transfected cells (Fig. 8C).

Aiming at providing evidence that the evaluated transfection activity and efficiency was essentially confined to neuronal cells, immunocytochemical characterization of cell cultures was performed. For this purpose, primary hippocampal and cortical cells were characterized using antiMAP2, anti-GFAP, and anti-CD11b antibodies to specifically stain neurons, astrocytes, and microglia, respectively. Control staining, in the absence of transfection, showed that cultures were mainly composed of neurons, although some astrocytes were visible in some samples (Figs. 9A(I, II), B(I, II)). GFP expression and MAP2 colocalization clearly revealed that primary neurons were transfected with the GFP transgene, whereas no colocalization was verified for GFAP (Figs. 9A(III), B(III)). Overall, these observations may suggest that $\mathrm{Tf}$-lipoplexes exhibit some tropism for neurons.

\section{Discussion}

Gene therapy is an emerging strategy for the treatment of several diseases and may offer a powerful tool for the protection against neuronal insults and degeneration. In the present work, we addressed studies aiming at identifying parameters affecting transfection activity of lipoplexes in C6 glioma cells and in primary hippocampal and cortical neurons. The ultimate goal will be to generate lipid-based carriers that could be used for efficient brain gene delivery, either in in vivo or ex vivo protocols, thus constituting viable alternatives to viral vectors.

Considering the limited efficiency of cationic liposomes to mediate transfection of nondividing cells, such as neurons, and in an attempt to overcome this limitation, we evaluated whether the strategy previously developed in our laboratory, which includes association of $\mathrm{Tf}$ to the complexes, would lead to higher values of transfection. Coating of lipoplexes with $\mathrm{Tf}$ has been demonstrated to promote both the cellular uptake of lipoplexes (da Cruz et al., 2001) and to trigger cytoplasmic delivery of the carried DNA through destabilization of the endosomal membrane under acidic conditions (da Cruz et al., 2001; Simoes et al., 1998). Our results clearly indicate that such a strategy resulted in a significant enhancement of transfection in both neuronal primary cultures and cell line, this effect being dependent on the type of cells, net charge, and composition of the complexes. The enhancement of transfection activity, achieved upon association of $\mathrm{Tf}$, was particularly notorious for hippocampal neurons, for which the levels of luciferase gene expression mediated by plain lipoplexes are very low. In contrast, primary cultures of cortical neurons exhibited relatively high levels of transgene expression, even in the absence of Tf. The low level of luciferase expression in hippocampal neurons is probably due to a lower extent of lipoplex internalization and/or to the higher stage of cell differentiation when compared to cortical neurons. The possibility that the strength of the CMV promoter is dependent on the type of neurons should not be excluded, which may constitute an alternative explanation for the differences in transfection activity observed between hippocampal and cortical neurons. When Tf-lipoplexes were prepared at a 2/1 lipid/DNA (+/-) charge ratio, higher levels of gene expression were obtained, although the most dramatic effect, when compared to plain lipoplexes, was observed for the 1/2 and 1/1 lipoplexes. The Tf-lipoplexes used are known for their nonspecific receptor-mediated binding and endocytosis (Simoes et al., 1999b), which may be attributed to their structure. It should be emphasized that transferrin is not covalently linked to the lipoplexes but is electrostatically associated to the cationic liposomes. Therefore, not all the $\mathrm{Tf}$ binding motifs may be available at the surface of the lipoplexes to bind to its receptors on the target cells. Consequently, this strategy is significantly different from other approaches involving the coupling of monoclonal antibodies against the Tf receptor (TfRMAb) (Shi et al., 2001). Although the transfection efficiency mediated by Tf-lipoplexes was significantly lower as compared to that achieved with the strategy involving the use of the TfRMAb, the risk of immunogenicity is low since no antibodies are used. Moreover, Tf-lipoplexes may constitute a valuable tool if only a restricted brain area is aimed at being transfected, as suggested from our preliminary data (not shown) involving their stereotactic administration into the rat striatum. The tailoring of the lipoplexes, in terms of their physicochemical properties, as a function of the cell 
type, is therefore of crucial importance for the optimization of lipid-based vectors.

Moreover, our results showed that liposome composition also plays a role in the transfection of neuronal cells mediated by lipoplexes, those composed of DOTAP:Chol being the most efficient. This reinforces the idea that inclusion of Chol in a cationic liposome formulation, such as that containing DOTAP, can enhance gene transfer to target cells in vitro and mainly in vivo. The high stability of Chol-containing lipoplexes (as compared to no Chol-containing lipoplexes) and their reduced binding ability to polyanionic components like serum proteins (Faneca et al., 2002) may contribute to this enhancing effect.

Cellular distribution of GFP expression was examined for the experimental conditions that led to the highest levels of transfection activity (as assessed by luciferase gene expression). Although a low percentage of GFP positive hippocampal and cortical neurons was observed (4\% for Tfassociated lipoplexes), it represents a significant increase when compared to plain lipoplexes. Although a significant increase in both transfection activity and efficiency was observed, as compared to values reported by others for hippocampal neurons (Kaech et al., 1996), the most remarkable values were found for cortical neurons. Moreover, Tflipoplexes appear to exhibit a neuronal tropism, since transfection was confined to neurons although astrocytes were also present in the cultures. Most of the studies concerning gene transfer into neuronal primary cultures have been focused on hippocampal neurons. Our results on cortical neurons are of particular interest, since the cerebral cortex is also a target region for the treatment of neuropathologies, such as Alzheimer's disease and ischemic insults.

In an attempt to gain insights into the mechanism of internalization of lipoplexes by neuronal cells, these were pretreated with metabolic inhibitors of endocytosis before transfection. An almost complete inhibition of transfection was observed, clearly showing that lipoplexes (both plain and Tf-associated) are internalized primarily via the endocytotic pathway, this being in agreement with the fact that clathrin-mediated membrane uptake in neurons is an important entry route for several molecules, such as neurotrophic toxins, and membrane receptors (De Camilli and Takei, 1996; Owen and Evans, 1998).

When testing the duration of luciferase gene expression, we observed that this was maximal at 12 and $24 \mathrm{~h}$ after transfection for C6 cells and primary hippocampal neurons, respectively. A rapid decrease in transgene expression was observed thereafter, indicating a short-term expression consistent with the fact that the plasmid remains episomal in the cell nucleus. This time dependence of gene expression may represent a problem if a sustained protein expression is required. However, for primary cortical neurons, this problem seems to be attenuated, since a relatively stable expression was observed up to 7 days post-transfection. These results, together with the fact that the treatment of some
CNS injuries may be achieved with a short-term expression of trophic proteins, show that Tf-associated lipoplexes may represent a valuable strategy for gene therapy of CNS acute and transient pathological processes.

It has been shown previously that the stage of cell maturation/differentiation may affect the transfection efficiency of postmitotic cells (Pelisek et al., 2001; Simoes et al., 1999c). The effect of the stage of differentiation on the levels of transgene expression in primary neurons was demonstrated upon transfection of the cells at different days over the culture period. At later stages of differentiation, a significant reduction of the levels of gene expression was observed. As a consequence of the nondividing nature of the cells, a higher stage of maturation and consequent decreased endocytotic activity may result in a reduction in the extent of internalization of the lipoplexes, thus leading to low levels of transfection. Therefore, the knowledge of the optimal transfection conditions for this type of cells may be of relevance if an ex vivo gene therapy approach is intended to be settled.

Altogether, our results show that many variables influence lipoplex-mediated transfection of neuronal cells. Our findings suggest that lipoplexes prepared upon association of $\mathrm{Tf}$ to cationic liposomes containing Chol, with adequate charge ratios, may prove to be a useful strategy for the delivery of therapeutic genes into neuronal cells.

\section{Acknowledgments}

This work was supported by (1) FCT, PRAXIS XXI (BD/ 19529/99) and (2) Grant FCT/BIO/36202/00 (Portugal).

\section{References}

Aiuti, A., Slavin, S., Aker, M., Ficara, F., Deola, S., Mortellaro, A., Morecki, S., Andolfi, G., Tabucchi, A., Carlucci, F., Marinello, E., Cattaneo, F., Vai, S., Servida, P., Miniero, R., Roncarolo, M.G., Bordignon, C., 2002. Correction of ADA-SCID by stem cell gene therapy combined with nonmyeloablative conditioning. Science 296, 2410-2413.

Banker, G.A., Cowan, W.M., 1977. Rat hippocampal neurons in dispersed cell culture. Brain Res. 126, 397-442.

Bartlett, G.R., 1959. Phosphorus assay in column chromatography. J. Biol. Chem. 254, 466-468.

Berry, M., Barrett, L., Seymour, L., Baird, A., Logan, A., 2001. Gene therapy for central nervous system repair. Curr. Opin. Mol. Ther. 3, $338-349$

Brewer, G.J., Torricelli, J.R., Evege, E.K., Price, P.J., 1993. Optimized survival of hippocampal neurons in B27-supplemented Neurobasal, a new serum-free medium combination. J. Neurosci. Res. 35, 567-576.

Cavazzana-Calvo, M., Hacein-Bey, S., de Saint Basile, S., Gross, F., Yvon, E., Nusbaum, P., Selz, F., Hue, C., Certain, S., Casanova, J.L., Bousso, P., Deist, F.L., Fischer, A., 2000. Gene therapy of human severe combined immunodeficiency (SCID)-X1 disease. Science 288, 669-672.

Chan, C.K., Jans, D.A., 1999. Enhancement of polylysine-mediated transferrinfection by nuclear localization sequences: polylysine does not function as a nuclear localization sequence. Hum. Gene Ther. 10, $1695-1702$.

Cheng, P.W., 1996. Receptor ligand-facilitated gene transfer-enhancement 
of liposome-mediated gene transfer and expression by transferrin. Hum. Gene Ther. 7, 275-282.

Clark, K.R., Sferra, T.J., Lo, W., Qu, G., Chen, R.J., Johnson, P.R., 1999. Gene transfer into the CNS using recombinant adeno-associated virus: analysis of vector DNA forms resulting in sustained expression. J. Drug Targeting 7, 269-283.

Crook, K., Stevenson, B.J., Dubouchet, M., Porteous, D.J., 1998. Inclusion of cholesterol in DOTAP transfection complexes increases the delivery of DNA to cells in vitro in the presence of serum. Gene Ther. 5, 137-143.

da Cruz, M.T., Simoes, S., Pires, P.P., Nir, S., de Lima, M.C., 2001. Kinetic analysis of the initial steps involved in lipoplex-cell interactions: effect of various factors that influence transfection activity. Biochim. Biophys. Acta $1510,136-151$

De Camilli, P., Takei, K., 1996. Molecular mechanisms in synaptic vesicle endocytosis and recycling. Neuron 16, 481-486.

de Lima, M.C.P., Simoes, S., Pires, P., Gaspar, R., Slepushkin, V., Duzgunes, N., 1999. Gene delivery mediated by cationic liposomes: from biophysical aspects to enhancement of transfection. Mol. Membr. Biol. 16, 103-109.

Faneca, H., Simoes, S., de Lima, M.C., 2002. Evaluation of lipid-based reagents to mediate intracellular gene delivery. Biochim. Biophys. Acta $1567,23-33$.

Farhood, H., Serbina, N., Huang, L., 1995. The role of dioleoyl phosphatidylethanolamine in cationic liposome mediated gene transfer. Biochim. Biophys. Acta 1235, 289-295.

Felgner, P.L., 1996. Improvements in cationic liposomes for in vivo gene transfer. Hum. Gene Ther. 7, 1791-1793.

Felgner, P.L., Gadek, T.R., Holm, M., Roman, R., Chan, H.W., Wenz, M., Northrop, J.P., Ringold, G.M., Danielsen, M., 1987. Lipofection: a highly efficient, lipid-mediated DNA-transfection procedure. Proc. Natl. Acad. Sci. U. S. A. 84, 7413-7417.

Felgner, J.H., Kumar, R., Sridhar, C.N., Wheeler, C.J., Tsai, Y.J., Border, R., Ramsey, P., Martin, M., Felgner, P.L., 1994. Enhanced gene delivery and mechanism studies with a novel series of cationic lipid formulations. J. Biol. Chem. 269, 2550-2561.

Fields, R.D., Lancaster, M.V., 1993. Dual-attribute continuous monitoring of cell proliferation/cytotoxicity. Am. Biotechnol. Lab. 11, 48-50.

Friend, D.S., Papahadjopoulos, D., Debs, R.J., 1996. Endocytosis and intracellular processing accompanying transfection mediated by cationic liposomes. Biochim. Biophys. Acta 1278, 41-50.

Gao, X., Huang, L., 1991. A novel cationic liposome reagent for efficient transfection of mammalian cells. Biochem. Biophys. Res. Commun. $179,280-285$

Gao, X., Huang, L., 1995. Cationic liposome-mediated gene transfer. Gene Ther. 2, 710-722.

Garcia, V.E., Sharma, S.C., 1998. Rescue of retinal ganglion cells from axotomy-induced apoptosis through TRK oncogene transfer. NeuroReport 9, 3165-3170.

Goula, D., Remy, J.S., Erbacher, P., Wasowicz, M., Levi, G., Abdallah, B., Demeneix, B.A., 1998. Size, diffusibility and transfection performance of linear PEI/DNA complexes in the mouse central nervous system. Gene Ther. 5, 712-717.

Hafez, I.M., Maurer, N., Cullis, P.R., 2001. On the mechanism whereby cationic lipids promote intracellular delivery of polynucleic acids. Gene Ther. 8, 1188-1196.

Hertz, E., Yu, A.C.H., Hertz, L., Juurlink, B.H.J., Schousboe, A., 1989. Preparation of primary cultures of mouse cortical neurons. In: Shahar, A., De Vellis, J., Vernadakis, A., Haber, B. (Eds.), A Dissection and Tissue Culture Manual of the Nervous System. Alan R. Liss Inc., New York, pp. $183-186$.

Hughes, S.M., Moussavi, H., Sauter, S.L., Davidson, B.L., 2002. Viralmediated gene transfer to mouse primary neural progenitor cells. Molec. Ther. 5, 16-24.

Hui, K.M., Sabapathy, T.K., Oei, A.A., Chia, T.F., 1994. Generation of allo-reactive cytotoxic $\mathrm{T}$ lymphocytes by particle bombardment-mediated gene transfer. J. Immunol. Methods 171, 147-155.
Imaoka, T., Date, I., Ohmoto, T., Yasuda, T., Tsuda, M., 1998. In vivo gene transfer into the adult mammalian central nervous system by continuous injection of plasmid DNA-cationic liposome complex. Brain Res. 780, $119-128$.

Kaech, S., Kim, J.B., Cariola, M., Ralston, E., 1996. Improved lipid-mediated gene transfer into primary cultures of hippocampal neurons. Brain Res. Mol. Brain Res. 35, 344-348.

Kichler, A., Mechtler, K., Behr, J.P., Wagner, E., 1997. Influence of membrane-active peptides on lipospermine/DNA complex mediated gene transfer. Bioconjugate Chem. 8, 213-221.

King, L.A., Mitrophanous, K.A., Clark, L.A., Kim, V.N., Rohll, J.B., Kingsman, A.J., Colello, R.J., 2000. Growth factor enhanced retroviral gene transfer to the adult central nervous system. Gene Ther. 7, $1103-1111$.

Kofler, P., Wiesenhofer, B., Rehrl, C., Baier, G., Stockhammer, G., Humpel, C., 1998. Liposome-mediated gene transfer into established CNS cell lines, primary glial cells, and in vivo. Cell Transplant 7, $175-185$.

Lee, K.D., Nir, S., Papahadjopoulos, D., 1993. Quantitative analysis of liposome-cell interactions in vitro: rate constants of binding and endocytosis with suspension and adherent $\mathrm{J} 774$ cells and human monocytes. Biochemistry 32, 889-899.

Legendre, J.Y., Szoka, F.C., 1992. Delivery of plasmid DNA into mammalian cell lines using $\mathrm{pH}$-sensitive liposomes: comparison with cationic liposomes. Pharm. Res. 9, 1235-1242.

Lemkine, G.F., Goula, D., Becker, N., Paleari, L., Levi, G., Demeneix, B.A., 1999. Optimisation of polyethylenimine-based gene delivery to mouse brain. J. Drug Targeting 7, 305-312.

Liu, Y., Liggitt, D., Zhong, W., Tu, G., Gaensler, K., Debs, R., 1995. Cationic liposome-mediated intravenous gene delivery. J. Biol. Chem. 270, 24864-24870.

Martino, G., Furlan, R., Galbiati, F., Poliani, P.L., Bergami, A., Grimaldi, L.M.E., Adorini, L., Comi, G., 1998. A gene therapy approach to treat demyelinating diseases using nonreplicative herpetic vectors engineered to produce cytokines. Mult. Scler. 4, 222-227.

Murray, K.D., Mcquillin, A., Stewart, L., Etheridge, C.J., Cooper, R.G., Miller, A.D., Gurling, H.M., 1999. Cationic liposome-mediated DNA transfection in organotypic explant cultures of the ventral mesencephalon. Gene Ther. 6, 190-197.

Olivier, J.C., Huertas, R., Lee, H.J., Calon, F., Pardridge, W.M., 2002. Synthesis of pegylated immunonanoparticles. Pharm. Res. 19, $1137-1143$.

Owen, D.J., Evans, P.R., 1998. A structural explanation for the recognition of tyrosine-based endocytotic signals. Science 282, 1327-1332.

Pelisek, J., Armeanu, S., Nikol, S., 2001. Quiescence, cell viability, apoptosis and necrosis of smooth muscle cells using different growth inhibitors. Cell Proliferation 34, 305-320.

Pires, P., Simoes, S., Nir, S., Gaspar, R., Duzgunes, N., de Lima, M.C.P., 1999. Interaction of cationic liposomes and their DNA complexes with monocytic leukemia cells. Biochim. Biophys. Acta 1418, 71-84.

Plank, C., Oberhauser, B., Mechtler, K., Koch, C., Wagner, E., 1994. The influence of endosome-disruptive peptides on gene transfer using synthetic virus-like gene transfer systems. J. Biol. Chem. 269, 12918-12924.

Reid, T., Galanis, E., Abbruzzese, J., Sze, D., Wein, L.M., Andrews, J., Randlev, B., Heise, C., Uprichard, M., Hatfield, M., Rome, L., Rubin, J., Kirn, D., 2002. Hepatic arterial infusion of a replication-selective oncolytic adenovirus (d11520): phase II viral immunologic, and clinical endpoints. Cancer Res. 62, 6070-6079.

Roessler, B.J., Davidson, B.L., 1994. Direct plasmid mediated transfection of adult murine brain cells in vivo using cationic liposomes. Neurosci. Lett. 167, 5-10.

Sato, H., Hattori, S., Kawamoto, S., Kudoh, I., Hayashi, A., Yamamoto, I., Yoshinari, M., Minami, M., Kanno, H., 2000. In vivo gene gun-mediated DNA delivery into rodent brain tissue. Biochem. Biophys. Res. Commun. 270, 163-170.

Schwartz, B., Benoist, C., Abdallah, B., Rangara, R., Hassan, A., Scher- 
man, D., Demeneix, B.A., 1996. Gene transfer by naked DNA into adult mouse brain. Gene Ther. 3, 405-411.

Sedmak, J.J., Grossberg, S.E., 1977. A rapid, sensitive, and versatile assay for protein using Coomassie brilliant blue G250. Anal. Biochem. 79, $544-552$.

Shi, N., Boado, R.J., Pardridge, W.M., 2001. Receptor-mediated gene targeting to tissues in vivo following intravenous administration of pegylated immunoliposomes. Pharm. Res. 18, 1091-1095.

Simoes, S., Slepushkin, V., Gaspar, R., de Lima, M.C.P., Duzgunes, N., 1998. Gene delivery by negatively charged ternary complexes of DNA, cationic liposomes and transferrin or fusigenic peptides. Gene Ther. 5, 955-964.

Simoes, S., Slepushkin, V., Gaspar, R., de, M.C., Duzgunes, N., 1999. Successful transfection of lymphocytes by ternary lipoplexes. Biosci. Rep. 19, 601-609.

Simoes, S., Slepushkin, V., Pires, P., Gaspar, R., de Lima, M.C.P., Duzgunes, N., 1999. Mechanisms of gene transfer mediated by lipoplexes associated with targeting ligands or $\mathrm{pH}$-sensitive peptides. Gene Ther. 6 , $1798-1807$.

Simoes, S., Slepushkin, V., Pretzer, E., Dazin, P., Gaspar, R., de Lima, M.C.P., Duzgunes, N., 1999. Transfection of human macrophages by lipoplexes via the combined use of transferrin and $\mathrm{pH}$-sensitive peptides. J. Leukoc. Biol. 65, 270-279.

Simoes, S., Slepushkin, V., Pires, P., Gaspar, R., de Lima, M.C.P., Duzgunes, N., 2000. Human serum albumin enhances DNA transfection by lipoplexes and confers resistance to inhibition by serum. Biochim. Biophys. Acta $1463,459-469$.

Slepushkin, V.A., Simoes, S., Dazin, P., Newman, M.S., Guo, L.S., de Lima, M.C.P., Duzgunes, N., 1997. Sterically stabilized pH-sensitive liposomes-Intracellular delivery of aqueous contents and prolonged circulation in vivo. J. Biol. Chem. 272, 2382-2388.

Stegmann, T., Legendre, J.Y., 1997. Gene transfer mediated by cationic lipids-Lack of a correlation between lipid mixing and transfection. Biochim. Biophys. Acta 1325, 71-79.
Templeton, N.S., Lasic, D.D., Frederik, P.M., Strey, H.H., Roberts, D.D., Pavlakis, G.N., 1997. Improved DNA-liposome complexes for increased systemic delivery and gene expression. Nat. Biotech. 15, $647-652$

Thomas, A., Kim, D.S., Fields, R.L., Chin, H., Gainer, H., 1998. Quantitative analysis of gene expression in organotypic slice-explant cultures by particle-mediated gene transfer. J. Neurosci. Methods 84, 181-191.

Wagner, E., Curiel, D., Cotten, M., 1994. Delivery of drugs, proteins and genes into cells using transferrin as a ligand for receptor-mediated endocytosis. Adv. Drug Delivery Rev. 14, 113-135.

White, M.J., DiCaprio, M.J., Greenberg, D.A., 1996. Assessment of neuronal viability with Alamar blue in cortical and granule cell cultures. J. Neurosci. Methods 70, 195-200.

Wu, B.Y., Liu, R.Y., So, K.L., Yu, A.C.H., 2000. Multi-lipofection efficiently transfected genes into astrocytes in primary culture. J. Neurosci. Methods 102, 133-141.

Xu, Y., Szoka, F.C.J., 1996. Mechanism of DNA release from cationic liposome/DNA complexes used in cell transfection. Biochemistry 35 , $5616-5623$.

Zabner, J., Fasbender, A.J., Moninger, T., Poellinger, K.A., Welsh, M.J., 1995. Cellular and molecular barriers to gene transfer by a cationic lipid. J. Biol. Chem. 270, 18997-19007.

Zanta, M.A., Belguise-Valladier, P., Behr, J.P., 1999. Gene delivery: a single nuclear localization signal peptide is sufficient to carry DNA to the cell nucleus. Proc. Natl. Acad. Sci. U. S. A. 96, 91-96.

Zhou, X., Huang, L., 1994. DNA transfection mediated by cationic liposomes containing lipopolylysine: characterization and mechanism of action. Biochim. Biophys. Acta 1189, 195-203.

Zhu, N., Liggitt, D., Liu, Y., Debs, R., 1993. Systemic gene expression after intravenous DNA delivery into adult mice. Science 261, 209-211.

Zou, L.L., Huang, L., Hayes, R.L., Black, C., Qiu, Y.H., Perez-Polo, J.R., Le, W., Clifton, G.L., Yang, K., 1999. Liposome-mediated NGF gene transfection following neuronal injury: potential therapeutic applications. Gene Ther. 6, 994-1005. 